\title{
Assessing the Individual in Team Based Design Projects
}

\author{
Roger Carrick and Alex Czekanski. \\ Department of Mechanical Engineering, York University \\ roger.carrick@lassonde.yorku.ca, alex.czekanski@lassonde.yorku.ca
}

\begin{abstract}
Project-based learning (PBL) has become a common practice engineering schools, often used in the context of design projects. Team based design projects allow the assessment of a broad range of graduate attributes, such as teamwork, communication, professionalism, ethics, project management, problem solving and design. Assessment of these skills is often qualitative, making assessment more difficult and varied than technical, quantitatively assessed subjects.
\end{abstract}

Most often when we grade the outputs of team-based projects, we assess the team as a whole; assigning one grade to the entire team. Whether it is project reports, formative and summative, presentations or group assignments, team members share a mark. Tools such as peer and self-evaluations and contribution attestations are sometimes used to modify the marks assigned to individuals, relating the relative engagement of students within the team, but they do not clearly link the direct learning outcomes of individuals to specific attributes. Shared grading is done for several reasons. Logistically, it is significantly less workload to mark a single report per group, than to mark individual reports. Second, in professional work, the output of a team is what is important, and is the primary indicator of success. In an academic environment however, it is the specific learning outcomes of the individuals that we wish to assess.

Understanding the learning outcomes of individuals in these team environments is important both from an accreditation standpoint, but also from a student motivation standpoint. One of the primary drivers for student learning is assessment. Additionally, for students to effectively work in a team, there are several key behaviors that need to be encouraged. These include interdependence, and trust; negative behaviors such as social loafing should be avoided. By implementing individual assessment, we believe issues related to social loafing can be reduced, and communication between team members improved.

In this work, we will look at the implementation of two individual assessment techniques, design journals and oral examinations, into a design project course that previously was assessed primarily on the output of teams. Students were surveyed on their reaction to the implementation of the individual assessments, as were course directors, and accreditation administration. Additionally, the effectiveness of these techniques in improving the team cohesiveness and reducing social loafing were examined by comparing the results of student peer evaluations with previous iterations of this course. The results showed that communication was improved, but there was a significant increase in the workload of course directors and teaching assistants. 